\title{
Non-equilibrium thermodynamic study of magnetization dynamics in the presence of spin-transfer torque
}

\author{
Kazuhiko Seki and Hiroshi Imamura \\ Nanotechnology Research Institute, National Institute of Advanced Industrial Science and Technology, \\ 1-1-1 Umezono, Tsukuba, Ibaraki 305-8568, Japan
}

\begin{abstract}
The dynamics of magnetization in the presence of spin-transfer torque was studied. We derived the equation for the motion of magnetization in the presence of a spin current by using the local equilibrium assumption in non-equilibrium thermodynamics. We show that, in the resultant equation, the ratio of the Gilbert damping constant, $\alpha$, and the coefficient, $\beta$, of the current-induced torque, called non-adiabatic torque, depends on the relaxation time of the fluctuating field $\tau_{c}$. The equality $\alpha=\beta$ holds when $\tau_{c}$ is very short compared to the time scale of magnetization dynamics. We apply our theory to current-induced magnetization reversal in magnetic multilayers and show that the switching time is a decreasing function of $\tau_{c}$.
\end{abstract}

Spin-transfer torque-induced magnetization dynamics such as current-induced magnetization reversal [1, 2, 3], domain wall motion [4], and microwave generation [5] have attracted a great deal of attention because of their potential applications to future nano-spinelectronic devices. In the absence of spin-transfer torque, magnetization dynamics is described by either the Landau-Lifshitz (LL) equation [6] or the Landau-Lifshitz-Gilbert (LLG) equation [7]. It is known that the LL and LLG equations become equivalent through rescaling of the gyromagnetic ratio.

However, this is not the case in the presence of spintransfer torque. For domain wall dynamics, the following LLG-type equation has been studied by several groups $[8,9,10]$ :

$$
\begin{aligned}
& \partial_{t}\langle\boldsymbol{M}\rangle+\boldsymbol{v} \cdot \boldsymbol{\nabla}\langle\boldsymbol{M}\rangle=\gamma \boldsymbol{H} \times\langle\boldsymbol{M}\rangle \\
& +\frac{\alpha}{M}\langle\boldsymbol{M}\rangle \times \partial_{t}\langle\boldsymbol{M}\rangle+\frac{\beta}{M}\langle\boldsymbol{M}\rangle \times[(\boldsymbol{v} \cdot \boldsymbol{\nabla})\langle\boldsymbol{M}\rangle],
\end{aligned}
$$

where $\boldsymbol{M}$ represents the magnetization, $\boldsymbol{v}$ is the velocity, $\gamma$ is the gyromagnetic ratio and $\alpha$ is the Gilbert damping constant. The second term on the left-hand side represents the adiabatic contribution of spin-transfer torque. The first and the second terms on the right-hand side are the torque due to the effective magnetic field $\boldsymbol{H}$ and the Gilbert damping. The last term on the right-hand side of Eq. (11) represents the current-induced torque, called "non-adiabatic torque" or simply the $\beta$ term. The directions of the adiabatic contribution of spin-transfer torque and non-adiabatic torque are shown in Fig. 1 (a).

As shown by Thiaville et al., the value of the coefficient $\beta$ strongly influences the motion of the domain wall [8]. However, the value of the coefficient $\beta$ is still controversial, and different conclusions have been drawn from different approaches [9, 10, 11, 12, 13, 14, 15]. For example, Barnes and Maekawa showed that the value of $\beta$ should be equal to that of the Gilbert damping constant $\alpha$ to satisfy the requirement that the relaxation should cease at the minimum of electrostatic energy, even under particle flow. Kohno et al. performed microscopic calculations (a)

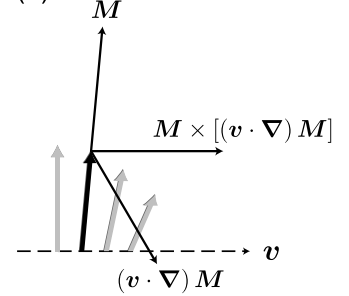

(b)

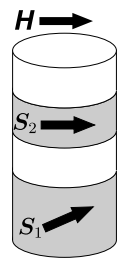

(c)

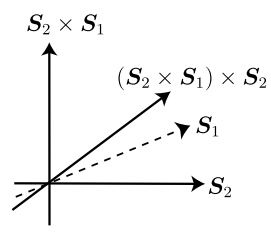

FIG. 1: (a) The direction of the magnetization $\boldsymbol{M}$, the adiabatic contribution of spin-transfer torque, $(\boldsymbol{v} \cdot \boldsymbol{\nabla}) \boldsymbol{M}$, and the $\beta$ term, $\boldsymbol{M} \times[(\boldsymbol{v} \cdot \boldsymbol{\nabla}) \boldsymbol{M}]$, are shown. The direction of the velocity $\boldsymbol{v}$ is indicated by the dotted arrow. (b) The magnetic multilayers, in which the pinned and the free layers are separated by a nonmagnetic spacer layer are schematically shown. The magnetization vectors of the pinned and free layers are represented by $\boldsymbol{S}_{1}$ and $\boldsymbol{S}_{s}$, respectively. The effective magnetic field to which $\boldsymbol{S}_{2}$ is subject is represented by $\boldsymbol{H}$. (c) The direction of the magnetization of the free layer, $\boldsymbol{S}_{2}$, the spin-transfer torque $\left(\boldsymbol{S}_{2} \times \boldsymbol{S}_{1}\right) \times \boldsymbol{S}_{2}$, and the non-adiabatic torque, $\boldsymbol{S}_{2} \times \boldsymbol{S}_{1}$, are shown. The direction of $\boldsymbol{S}_{1}$ is indicated by the dotted arrow.

of spin torques in disordered ferromagnets and showed that the $\alpha$ and $\beta$ terms arise from the spin relaxation processes and that $\alpha \neq \beta$ in general [10]. Tserkovnyak et al. [11] derived the $\beta$ term using a quasiparticle approximation and showed that $\alpha=\beta$ within a self-consistent picture based on the local density approximation.

In the current-induced magnetization dynamics in the magnetic multilayers shown in Fig. 1(b) [16, 17, 18], the non-adiabatic torque exerts a strong effect, and therefore affects the direct-current voltage of the spin torque diode, as shown in Refs. [17, 18]. The magnetization dynamics of the free layer, $\boldsymbol{S}_{2}$, has been studied by using the following LLG-type equation,

$$
\begin{aligned}
& \partial_{t} \boldsymbol{S}_{2}-\frac{I}{e} g \hbar\left(\boldsymbol{S}_{2} \times \boldsymbol{S}_{1}\right) \times \boldsymbol{S}_{2}=\gamma \boldsymbol{H} \times \boldsymbol{S}_{2}+\frac{\alpha}{S_{2}} \boldsymbol{S}_{2} \times \partial_{t} \boldsymbol{S}_{2} \\
& +\eta I \boldsymbol{S}_{2} \times \boldsymbol{S}_{1},
\end{aligned}
$$

where $I$ is the charge current density, $g$ is the amplitude of the spin torque introduced by Slonczewski [1], $\hbar$ is 
the Dirac constant and $\eta$ represents the magnitude of the "non-adiabatic torque" which is sometimes called the field-like torque [17, 18].

In this paper, we study the magnetization dynamics induced by spin-transfer torque in the framework of nonequilibrium thermodynamics. We derive the equation of motion of the magnetization in the presence of a spin current by using the local equilibrium assumption. In the resultant equation, the Gilbert damping term and the $\beta$ term are expressed as memory terms with the relaxation time of the fluctuating field $\tau_{c}$. We show that the value of the coefficient $\beta$ is not equal to that of the Gilbert damping constant $\alpha$ in general. However, we also show that the equality $\alpha=\beta$ holds if $\tau_{c} \ll 1 /(\gamma H)$. We apply our theory to the current-induced magnetization reversal in magnetic multilayers and show that the switching time is a decreasing function of $\tau_{c}$.

Let us first briefly introduce the non-equilibrium statistical theory of magnetization dynamics in the absence of spin current [19]. The LLG equation describing the motion of magnetization $\boldsymbol{M}$ under an effective magnetic field $\boldsymbol{H}$ is given by

$$
\partial_{t} \boldsymbol{M}=\gamma \boldsymbol{H} \times \boldsymbol{M}+\frac{\alpha}{M} \boldsymbol{M} \times \partial_{t} \boldsymbol{M}
$$

The equivalent LL equation is expressed as

$$
\partial_{t} \boldsymbol{M}=\frac{\gamma}{1+\alpha^{2}} \boldsymbol{H} \times \boldsymbol{M}-\frac{\alpha \gamma}{M\left(1+\alpha^{2}\right)} \boldsymbol{M} \times(\boldsymbol{M} \times \boldsymbol{H}) .
$$

The Langevin equations leading to Eqs. (3) and (4) by taking the ensemble average of magnetization $\boldsymbol{m}$, are

$$
\begin{aligned}
& \partial_{t} \boldsymbol{m}=\gamma \boldsymbol{H}_{\mathrm{tot}} \times \boldsymbol{m} \\
& \partial_{t} \delta \boldsymbol{H}=-\frac{1}{\tau_{c}}\left(\delta \boldsymbol{H}-\chi_{s} \boldsymbol{m}\right)+\boldsymbol{R}(t),
\end{aligned}
$$

where the total magnetic field $\boldsymbol{H}_{\text {tot }}$ is the sum of the effective magnetic field $\boldsymbol{H}$ and the fluctuating magnetic field $\delta \boldsymbol{H}$ and $\chi_{s}$ is the susceptibility of the local magnetic field induced at the position of the spin. According to Eq. (6) the fluctuating magnetic field $\delta \boldsymbol{H}$ relaxes toward the reaction field $\chi_{s} \boldsymbol{m}$ with the relaxation time $\tau_{c}$. The random field $\boldsymbol{R}(t)$ satisfies $\langle\boldsymbol{R}(t)\rangle=0$ and the fluctuationdissipation relation, $\left\langle R_{i}(t) R_{j}\left(t^{\prime}\right)\right\rangle=\frac{2}{\tau_{c}} \chi_{s} k_{\mathrm{B}} T \delta_{i, j} \delta\left(t-t^{\prime}\right)$, where $k_{\mathrm{B}}$ is the Boltzmann constant, $T$ is the temperature, $\langle\cdots\rangle$ denotes the ensemble average, and $i, j=1,2,3$ represents the Cartesian components. It was shown that Eqs. (5) and (6) lead to Kawabata's extended LandauLifshitz equation [20] derived by the projection operator method [19]. In the Markovian limit, i.e., $\tau_{c} \ll 1 /(\gamma H)$, we can obtain the LLG equation (3) and the corresponding LL equation (44) with $\alpha=\gamma \tau_{c} \chi_{s} M$ [19].

In order to consider the flow of spins, i.e., spin current, we introduce the positional dependence. Since we are interested in the average motion, it is convenient to introduce the mean velocity of the carrier, $\boldsymbol{v}$. The average magnetization, $\langle\boldsymbol{m}(\boldsymbol{x}, t)\rangle$, is obtained by introducing the positional dependence and taking the ensemble average of Eq. (5). In terms of the mean velocity, the ensemble average of the left-hand side of Eq. (5) leads to

$$
\partial_{t}\langle\boldsymbol{m}\rangle+(\boldsymbol{v} \cdot \boldsymbol{\nabla})\langle\boldsymbol{m}\rangle .
$$

Assuming $\langle\delta \boldsymbol{H} \times \boldsymbol{m}\rangle \approx\langle\delta \boldsymbol{H}\rangle \times\langle\boldsymbol{m}\rangle$, which is applicable when the thermal fluctuation is small compared to the mean value, we obtain

$$
\partial_{t}\langle\boldsymbol{m}\rangle+(\boldsymbol{v} \cdot \boldsymbol{\nabla})\langle\boldsymbol{m}\rangle=\gamma\left\langle\boldsymbol{H}_{\mathrm{tot}}(\boldsymbol{x}, t)\right\rangle \times\langle\boldsymbol{m}(\boldsymbol{x}, t)\rangle .
$$

The mean magnetization density is expressed as $\langle\boldsymbol{M}(\boldsymbol{x}, t)\rangle=\rho(\boldsymbol{x}, t)\langle\boldsymbol{m}(\boldsymbol{x}, t)\rangle$, i.e., by the product of the scalar and vectorial components both of which depend on the position of the spin carrier at time $t$. The spin carrier density satisfies the continuity equation,

$$
\partial_{t} \rho(\boldsymbol{x}, t)+\nabla \cdot(\boldsymbol{v} \rho(\boldsymbol{x}, t))=0 .
$$

By multiplying the left-hand side of Eq. (8) by $\rho(\boldsymbol{x}, t)$ and using the continuity equation (9), the closed expression for the mean magnetization is obtained as [21]

$$
\begin{aligned}
\rho\left(\partial_{t}\langle\boldsymbol{m}\rangle+\boldsymbol{v} \cdot \boldsymbol{\nabla}\langle\boldsymbol{m}\rangle\right) & =\partial_{t} \rho\langle\boldsymbol{m}\rangle+\langle\boldsymbol{m}\rangle \boldsymbol{\nabla} \cdot \boldsymbol{v} \rho+\rho \boldsymbol{v} \cdot \boldsymbol{\nabla}\langle\boldsymbol{m}\rangle \\
& =\partial_{t}\langle\boldsymbol{M}\rangle+\operatorname{Div} \boldsymbol{v}\langle\boldsymbol{M}\rangle
\end{aligned}
$$

where $\operatorname{Div} \boldsymbol{v}\langle\boldsymbol{M}\rangle$ is defined by

$$
\operatorname{Div} \boldsymbol{v}\langle\boldsymbol{M}\rangle=\sum_{i=1}^{3} \frac{\partial v_{i}\langle\boldsymbol{M}\rangle}{\partial x_{i}}=\langle\boldsymbol{M}\rangle(\boldsymbol{\nabla} \cdot \boldsymbol{v})+(\boldsymbol{v} \cdot \boldsymbol{\nabla})\langle\boldsymbol{M}\rangle .(11)
$$

By multiplying the right-hand side of Eq. (8) by $\rho(\boldsymbol{x}, t)$ and using Eq. (10), we obtain

$$
\partial_{t}\langle\boldsymbol{M}\rangle+\operatorname{Div} \boldsymbol{v}\langle\boldsymbol{M}\rangle=\gamma(\boldsymbol{H}+\langle\delta \boldsymbol{H}\rangle) \times\langle\boldsymbol{M}\rangle .
$$

Equation (12) takes the standard form of a timeevolution equation for extensive thermodynamical variables under flow [21]. The average of Eq. (6) with the positional dependence is given by

$$
\partial_{t}\langle\delta \boldsymbol{H}(\boldsymbol{x}, t)\rangle=-\frac{1}{\tau_{c}}[\langle\delta \boldsymbol{H}(\boldsymbol{x}, t)\rangle-\chi\langle\boldsymbol{M}(\boldsymbol{x}(t), t)\rangle],
$$

where $\boldsymbol{x}(t)$ is the mean position at time $t$ of the spin carrier, which flows with velocity $\boldsymbol{v}=\partial_{t} \boldsymbol{x}(t)$ and $\chi=\chi_{s} / \rho$ is assumed to be a constant independent of the position. Equations (12) and (13) constitute the basis for the subsequent study of magnetization dynamics in the presence of spin-transfer torque.

The formal solution of Eq. (13) is expressed as

$$
\langle\delta \boldsymbol{H}(\boldsymbol{x}, t)\rangle=\frac{\chi}{\tau_{c}} \int_{-\infty}^{t} \psi\left(t-t^{\prime}\right)\left\langle\boldsymbol{M}\left(\boldsymbol{x}\left(t^{\prime}\right), t^{\prime}\right)\right\rangle d t^{\prime},
$$

where the memory kernel is given by $\psi(t)=\exp \left[-t / \tau_{c}\right]$. Using partial integration, we obtain

$$
\langle\delta \boldsymbol{H}(\boldsymbol{x}, t)\rangle=\chi\langle\boldsymbol{M}\rangle-\int_{-\infty}^{t} \psi\left(t-t^{\prime}\right) \chi\left\langle\dot{\boldsymbol{M}}\left(t^{\prime}\right)\right\rangle d t^{\prime},
$$


where the explicit expression for $\dot{\boldsymbol{M}}(t)=\dot{\boldsymbol{M}}(\boldsymbol{x}(t), t)$ is given by the convective derivative,

$$
\dot{\boldsymbol{M}}(t)=\partial_{t} \boldsymbol{M}(\boldsymbol{x}(t), t)+(\boldsymbol{v} \cdot \boldsymbol{\nabla}) \boldsymbol{M}(\boldsymbol{x}(t), t) .
$$

Substituting Eq. (15) into Eq. (12), we obtain the equation of motion for the mean magnetization density,

$$
\begin{aligned}
& \partial_{t}\langle\boldsymbol{M}\rangle+\operatorname{Div} \boldsymbol{v}\langle\boldsymbol{M}\rangle=\gamma \boldsymbol{H} \times\langle\boldsymbol{M}\rangle \\
& +\gamma \int_{-\infty}^{t} d t^{\prime} \psi\left(t-t^{\prime}\right) \chi\langle\boldsymbol{M}(t)\rangle \times\left\langle\dot{\boldsymbol{M}}\left(t^{\prime}\right)\right\rangle .
\end{aligned}
$$

Equation (17) supplemented by Eq. (16) is the principal result of this paper.

When the relaxation time of the fluctuating field, $\tau_{c}$, is very short compared to the time scale of the magnetization dynamics, the memory kernel is decoupled and Eq. (17) can be written in the form of an LLG-type equation as

$$
\partial_{t}\langle\boldsymbol{M}\rangle+\operatorname{Div} \boldsymbol{v}\langle\boldsymbol{M}\rangle=\gamma \boldsymbol{H} \times\langle\boldsymbol{M}\rangle+\frac{\alpha}{M}\langle\boldsymbol{M}\rangle \times\langle\dot{\boldsymbol{M}}\rangle,
$$

where $\alpha=\gamma \tau_{c} \chi M$ is the Gilbert damping constant. Substituting the explicit form of the convective derivative, Eq. (16), into Eq. (18) and using Eq.(11) we obtain the following LLG-type equation:

$$
\begin{gathered}
\partial_{t}\langle\boldsymbol{M}\rangle+\langle\boldsymbol{M}\rangle(\boldsymbol{\nabla} \cdot \boldsymbol{v})+(\boldsymbol{v} \cdot \boldsymbol{\nabla})\langle\boldsymbol{M}\rangle=\gamma \boldsymbol{H} \times\langle\boldsymbol{M}\rangle \\
+\frac{\alpha}{M}\langle\boldsymbol{M}\rangle \times \partial_{t}\langle\boldsymbol{M}\rangle+\frac{\alpha}{M}\langle\boldsymbol{M}\rangle \times[(\boldsymbol{v} \cdot \boldsymbol{\nabla})\langle\boldsymbol{M}\rangle] .
\end{gathered}
$$

If $\boldsymbol{\nabla} \cdot \boldsymbol{v}=0$, Eq. (19) reduces to Eq. (14) of Ref. [9], which is derived by replacing the time derivative of magnetization $\partial_{t} \boldsymbol{M}$ on both sides of the LLG equation (3) by the convective derivative $\partial_{t} \boldsymbol{M}+\boldsymbol{v} \cdot \boldsymbol{\nabla} \cdot \boldsymbol{M}$. The term $\langle\boldsymbol{M}\rangle(\boldsymbol{\nabla} \cdot \boldsymbol{v})$ appears not on the right-hand side of Eq. (19) but on the left-hand side, which means we cannot obtain Eq. (19) using the same procedure used in Ref. [9]. As shown in Refs. 9, 22], Eq. (19) with $\langle\boldsymbol{M}\rangle(\boldsymbol{\nabla} \cdot \boldsymbol{v})=0$ leads to a steady-state solution in the comoving frame, $\langle\boldsymbol{M}(t)\rangle=\left\langle\boldsymbol{M}_{0}(\boldsymbol{x}-\boldsymbol{v} t)\right\rangle$, where $\left\langle\boldsymbol{M}_{0}(\boldsymbol{x})\right\rangle$ denotes the stationary solution in the absence of domain wall motion. However, if $\langle\boldsymbol{M}\rangle(\boldsymbol{\nabla} \cdot \boldsymbol{v}) \neq 0$, the steady-state solution may break the Galilean invariance. The situation $\langle\boldsymbol{M}\rangle(\boldsymbol{\nabla} \cdot \boldsymbol{v}) \neq 0$ can be realized, for example, in magnetic semiconductors [23, 24], where the spin carrier density is spatially inhomogeneous, i.e., $\nabla \rho \neq 0$.

The last term of Eq. (19) represents the non-adiabatic component of the current-induced torque, which is also known as the " $\beta$ term". By comparing Eq. (19) with Eq. (1), one can see that the coefficient of the last term is equal to the Gilbert damping constant $\alpha$. However, Eq. (19) is valid when the relaxation time of the fluctuating field, $\tau_{c}$, is very short compared to the time scale of the magnetization dynamics. It should be noted that the general form of the equation describing the magnetization dynamics is given by Eq. (17) where the last term on the right-hand side is the origin of the $\alpha$ and $\beta$ terms. It is possible to project the torque represented by the memory function onto the direction of the $\alpha$ and $\beta$ terms. This projection leads to $\alpha \neq \beta$ in general.

In order to observe the effect of $\tau_{c}$ on the magnetization dynamics we applied our theory to the currentinduced magnetization switching in the magnetic multilayer shown in Fig:1(b). We assumed that the fixed and free layers are single-domain magnetic layers acting as a large spin characterized by the total magnetization vector defined as $\boldsymbol{S}_{i}=\int d V\left\langle\boldsymbol{M}_{i}\right\rangle$, where $i=1(2)$ for the fixed (free) layer and $\int d V$ denotes the volume integration over the fixed (free) layer. Both the magnetization vector of the fixed layer $\boldsymbol{S}_{1}$ and the effective magnetic field, $\boldsymbol{H}$, acting on the free layer lie in the plane.

Integrating Eqs. (12) and (13) over the volume of the free layer, we obtain the equations,

$$
\begin{aligned}
& \partial_{t} \boldsymbol{S}_{2}+\int d S \hat{\boldsymbol{n}} \cdot \boldsymbol{J}=\gamma(\boldsymbol{H}+\langle\delta \boldsymbol{H}\rangle) \times \boldsymbol{S}_{2}, \\
& \partial_{t}\langle\delta \boldsymbol{H}\rangle=-\frac{1}{\tau_{c}}\left(\langle\delta \boldsymbol{H}\rangle-\chi_{V} \boldsymbol{S}_{2}\right),
\end{aligned}
$$

where $\boldsymbol{J}=\boldsymbol{v} \otimes\langle\boldsymbol{M}\rangle$ is the spin current tensor $\int d S$ represents the surface integration over the free layer, $\hat{\boldsymbol{n}}$ is the unit normal vector of the surface, and $\chi_{V}=\chi / V$ is defined by the volume of the free layer $V$.

The same procedure used to derive Eq. (17) yields

$$
\begin{aligned}
& \partial_{t} \boldsymbol{S}_{2}+\int d S \hat{\boldsymbol{n}} \cdot \boldsymbol{J}=\gamma \boldsymbol{H} \times \boldsymbol{S}_{2} \\
& +\gamma \int_{-\infty}^{t} d t^{\prime} \psi\left(t-t^{\prime}\right) \chi_{V} \boldsymbol{S}_{2}(t) \times \partial_{t^{\prime}} \boldsymbol{S}_{2}\left(t^{\prime}\right),
\end{aligned}
$$

where $\psi(t)=\exp \left[-t / \tau_{c}\right]$.

When the relaxation time of the fluctuating field is short compared to the time scale of magnetization dynamics, the LLG-type equation in the presence of the spin-transfer torque is obtained as

$$
\partial_{t} \boldsymbol{S}_{2}+\int d S \hat{\boldsymbol{n}} \cdot \boldsymbol{J}=\gamma \boldsymbol{H} \times \boldsymbol{S}_{2}+\frac{\alpha}{S_{2}} \boldsymbol{S}_{2} \times \partial_{t} \boldsymbol{S}_{2},
$$

where $\alpha=\gamma \tau_{c} \chi_{V} S_{2}$. By introducing the conventional form of the spin-transfer torque [1], we obtain the following LLG-type equation:

$$
\partial_{t} \boldsymbol{S}_{2}-\frac{I}{e} g \hbar\left(\boldsymbol{S}_{2} \times \boldsymbol{S}_{1}\right) \times \boldsymbol{S}_{2}=\gamma \boldsymbol{H} \times \boldsymbol{S}_{2}+\frac{\alpha}{S_{2}} \boldsymbol{S}_{2} \times \partial_{t} \boldsymbol{S}_{2} .
$$

However, Eq. (24) is valid only when $\tau_{c}<1 /(\gamma H)$. As mentioned before, the torque represented by using the memory function generally has a component parallel to the non-adiabatic torque. In order to observe the effect of the non-adiabatic torque induced by the memory function on the magnetization dynamics, we performed numerical simulation using Eqs. (20) and (21). 


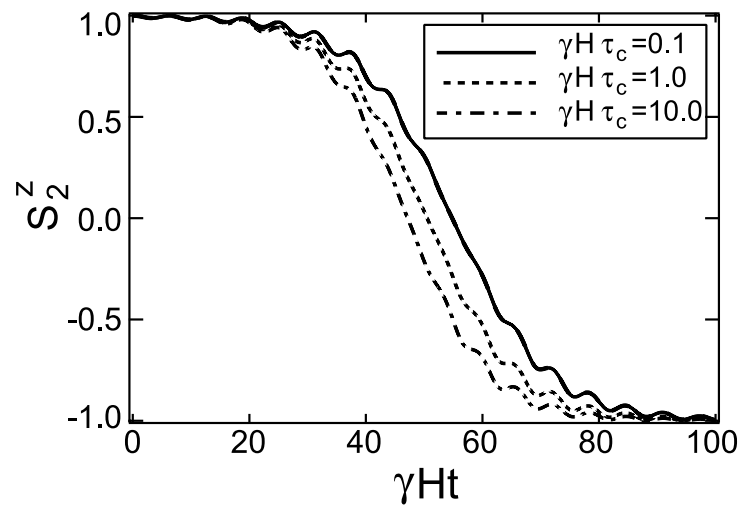

FIG. 2: The $z$-component of the magnetization $\boldsymbol{S}_{2}$ is plotted as a function of time for various values of $\tau_{c}$. The initial direction of the free layer is taken to lie in the direction of the effective magnetic field, which is aligned to the $z$ axis. The initial angle between $\boldsymbol{S}_{1}$ and $\boldsymbol{S}_{2}$ is taken to be $45^{\circ}$. The Gilbert damping constant $\alpha$ is 0.01 .

For the simulation, we used the following conditions. At the initial time of $t=0$, we assumed that the magnetization of the free layer is aligned parallel to the effective magnetic field $\boldsymbol{H}$ and the angle between the magnetizations of the fixed and the free layers is $45^{\circ}$. This arrangement corresponds to the recent experiment on a magnetic tunnel junction system [18]. We also assumed that the fluctuation field has zero mean value at $t=0$, i.e., $\langle\delta \boldsymbol{H}(0)\rangle=\mathbf{0}$.

In Fig. 2, we plot the time dependence of the $z$ component of the magnetization of the free layer, $\boldsymbol{S}_{2}$, under the large-enough spin current to flip the magnetization of the free layer, $I g \hbar S_{2}^{2} S_{1} /(e \alpha \gamma H)=-10$. The value of $\tau_{c}$ is varied while the value of $\alpha=0.01$ is maintained. The solid, dotted, and dot-dashed lines correspond to $\gamma H \tau_{c}=0.1,1.0$, and 10.0, respectively. As shown in Fig. 2 the time required for the magnetization of the free layer to flip decreases with increasing $\tau_{c}$, which can be understood by considering the non-adiabatic torque induced by the spin current. The non-adiabatic torque induced by the spin current is obtained by projecting the torque given by the last term of Eq. (22) onto the direction of $\boldsymbol{S}_{2} \times \boldsymbol{S}_{1}$, which results in the positive contribution to the spin-flip motion of $\boldsymbol{S}_{2}$. Since the last term of Eq. (22) includes a memory function, the nonadiabatic torque induced by the spin current increases with increasing $\tau_{c}$. Therefore, the time required for $\boldsymbol{S}_{2}$ to flip decreases with increasing $\tau_{c}$. For $\gamma H \tau_{c}>10$ we observe no further decrease of the time required for $\boldsymbol{S}_{2}$ to flip because the memory function is an integral of the vector $\boldsymbol{S}_{2}(t) \times \partial_{t^{\prime}} \boldsymbol{S}_{2}\left(t^{\prime}\right)$ and the contributions from the memory at $t-t^{\prime} \gg 1 /(\gamma H)$ is eliminated.

In conclusion, we derived the equation for the motion of magnetization in the presence of a spin current by using the local equilibrium assumption in non-equilibrium thermodynamics. We demonstrated that the value of the coefficient $\beta$ is not equal to that of the Gilbert damping constant $\alpha$ in general. However, we also show that the equality $\alpha=\beta$ holds if $\tau_{c} \ll 1 /(\gamma H)$. We then applied our theory to current-induced magnetization reversal in magnetic multilayers and showed that the switching time is a decreasing function of $\tau_{c}$.

The authors would like to acknowledge the valuable discussions they had with S.E. Barnes, S. Maekawa, P. M. Levy, K. Kitahara, K. Matsushita, J. Sato and T. Taniguchi. This work was supported by NEDO.

[1] J. C. Slonczewski, J. Magn. Magn. Mater. 159, L1 (1996).

[2] L. Berger, Phys. Rev. B 54, 9353 (1996).

[3] J. A. Katine, F. J. Albert, R. A. Buhrman, E. B. Myers, and D. C. Ralph, Phys. Rev. Lett. 84, 3149 (2000).

[4] M. Kläui, C. A. F. Vaz, J. A. C. Bland, W. Wernsdorfer, G. Faini, E. Cambril, and L. J. Heyderman, Appl. Phys. Lett. 83, 105 (2003).

[5] S. I. Kiselev, J. C. Sankey, I. N. Krivorotov, N. C. Emley, R. J. Schoelkopf, R. A. Buhrman, and D. C. Ralph, Nature 425, 380 (2003).

[6] L. Landau and E. Lifshitz, Phys. Z. Sowjet. 8, 153 (1935).

[7] T. L. Gilbert, Armour Research Foundation Project No. A059, Supplementary Report, May 1, 1959; IEEE Trans. Magn. 40, 3443 (2004).

[8] A. Thiaville, Y. Nakatani, J. Miltat, and Y. Suzuki, Europhys. Lett. 69, 990 (2005).

[9] S. E. Barnes and S. Maekawa, Phys. Rev. Lett. 95, 107204 (2005).

[10] H. Kohno, G. Tatara, and J. Shibata, J. Phys. Soc. Japan 75, 113706 (2006).

[11] Y. Tserkovnyak, H. J. Skadsem, A. Brataas, and G. E. W. Bauer, Phys. Rev. B 74, 144405 (2006).

[12] Y. Tserkovnyak, A. Brataas, and G. E. Bauer, J. Magn. Magn. Mater 320, 1282 (2008).

[13] J. Xiao, A. Zangwill, and M. D. Stiles, Phys. Rev. B 73, 054428 (2006).

[14] M. D. Stiles, W. M. Saslow, M. J. Donahue, and A. Zangwill, Phys. Rev. B 75, 214423 (2007).

[15] R. A. Duine, A. S. Nunez, J. Sinova, and A. H. MacDonald, Phys. Rev. B 75, 214420 (2007).

[16] J. Zhang, P. M. Levy, S. Zhang, and V. Antropov, Phys. Rev. Lett. 93, 256602 (2004).

[17] A. A. Tulapurkar et al., Nature 438, 339 (2005).

[18] H. Kubota et al., Nature Physics 4, 37 (2008).

[19] K. Miyazaki and K. Seki, J. Chem. Phys. 108, 7052 (1998).

[20] A. Kawabata, Prog. Theor. Phys 48, 2237 (1972).

[21] S. R. de Groot and P. Mazur, Nonequilibrium thermodynamics (North-Holland, Amsterdam, 1962).

[22] Y. Tserkovnyak, A. Brataas, and G. E. Bauer, J. Magn. Magn. Mater 8, 153 (1935).

[23] H. Ohno, Science 281, 951 (1998).

[24] T. Dietl and H. Ohno, Materials Today 9, 18 (2006). 